\title{
SOLIDIFICATION OF SEMI-TRANSPARENT MATERIALS BY CONDUCTION AND RADIATION
}

\author{
I. S. HABIB \\ University of Michigan, Dearborn Campus, Dearborn, Michigan, U.S.A.
}

(Received 16 April 1971)

\section{NOMENCLATURE}

$c$, specific heat capacity:

$E_{n}$, the exponential integral:

$k$, thermal conductivity:

$n$, index of refraction:

$q_{r}$, radiative heat flux: $q_{r, 0}$, radiative heat flux at $x=0 ; \quad q_{r, s}$, radiative heat flux at $x=s$;

$s$ solid-liquid interface, "melt line":

$t$, time, integration variable;

$T$, temperature:

\section{Greek symbols}

$\alpha$, thermal diffusivity:

$\varepsilon$, surface emissivity:

$\lambda$. latent heat of freezing:

$\rho$, density: $\rho_{1}, \rho_{2}$ surface relectivities:

$\sigma, \quad$ Stefan-Boltzmann constant:

$\kappa$, absorption coefficient:

$\tau, \quad$ optical depth $=\kappa x ; \quad \tau_{0}=\kappa s$.

\section{INTRODUCTION}

Problems of heat transfer in which a medium undergoes a phase change during exposure to a thermal environment continue to receive appreciable attention. Because of the non-linear nature of these problems, most of the solutions which have been obtained involved the use of numerical or approximate analytical techniques [1-3]. Little study, if any, has been devoted to such problems in semi-transparent materials in which a substantial contribution to the process is due to heat transfer by radiation. Problems of this nature arises in the recrystallization of semi-transparent slabs accompanied by a latent heat of recrystallization. In the present note we employ the integral method to study the effect of the radiative heat transfer on the solidification rate and on the temperature distribution in the solid phase. It is felt that in the majority of cases it is useful to have an approximate solution which can describe, however qualitatively, the phenomena under examination.

\section{ANALYSIS}

The problem considered is the one dimensional solidifi- cation of a semi-transparent thick homogeneous and isotropic material with constant thermophysical and optical properties. A unique melt temperature $T_{2}$ is assumed to exist and the liquid phase is maintained at this temperature. The surface at $x=0$ is considered to be the cold wall at a constant temperature $T_{1}$ with an emissivity $\varepsilon_{1}$. The melt line has an emissivity $\varepsilon_{2}$. No significant change in density occurs during solidification. The formulation given here is valid for the analogous melting problem with obvious modifications only.

The energy equation for the problem can be written in the form

$$
\rho c \frac{\partial T}{\partial t}=\frac{\partial}{\partial x}\left[k \frac{\partial T}{\partial x}-q_{r}\right] \text { for } 0<x<s
$$

subject to the following conditions

$$
\begin{aligned}
& k \frac{\partial T}{\partial t}-q_{r}=\rho \lambda \frac{\mathrm{d} s}{\mathrm{~d} t} \quad \text { at } x=s \\
& T(0, t)=T_{1} \\
& T(s, t)=T_{2}
\end{aligned}
$$

Integrating equation (1) from $x=0$ to $x=s$ using equation (2a) results in the following equation referred to as the heat balance integral.

$$
\left(\frac{\lambda}{c}+T_{2}\right) \frac{\mathrm{d} s}{\mathrm{~d} t}=\frac{\mathrm{d} \theta}{\mathrm{d} t}+\alpha\left(\frac{\partial T}{\partial x}\right)_{x=0}-\frac{q_{r, 0}}{\rho c}
$$

where

$$
\theta=\int_{0}^{s} T \mathrm{~d} x
$$

If we let the temperature $T$ be represented by a second degree polynomial, then three conditions are necessary to find the constants. Equations (2b) and (2c) are two conditions and the third one is essentially equation (2a). But equation (2a) in its present form is not suitable because the coefficients in the polynomial would involve $\mathrm{ds} / \mathrm{d} t$. In turn, the heat balance integral, equation (3), would involve second order derivatives for $s(t)$ whereas there is only one initial condition for $s$, namely $s(0)=0$. To avoid this difficulty we differentiate equation $(2 \mathrm{c})$ with respect to time 
$\frac{\partial T}{\partial x} \frac{\mathrm{d} s}{\mathrm{~d} t}+\frac{\partial T}{\partial t}=0$

or

$$
\frac{\mathrm{d} s}{\mathrm{~d} t}=-\frac{\partial T / \partial t}{\partial T / \partial x}
$$

If we substitute (5b) in (2a) we obtain

$$
\left(\frac{\partial T}{\partial x}\right)^{2}=-\frac{\rho \lambda}{k} \frac{\partial T}{\partial t}+\frac{q_{r}}{k} \frac{\partial T}{\partial x} .
$$

From the differential equation for the problem, equation (1), we substitute for $\partial T / \partial t$ in equation (6) and obtain the third required condition as

$$
\left(\frac{\partial T}{\partial x}\right)_{x=s}^{2}=\left[-\frac{\lambda \partial^{2} T}{c} \frac{\lambda}{\partial x^{2}}+\frac{\lambda}{k c} \frac{\mathrm{d} q_{r}}{\mathrm{~d} x}+\frac{q_{r}}{k} \frac{\partial T}{\partial x}\right]_{x=s}
$$

If we represent the temperature distribution in the following form

$$
T_{2}-T=A(x-s)+B(x-s)^{2}
$$

then using equation (2b) and equation (7), $A$ and $B$ are determined from the following expressions

$$
\begin{gathered}
A=\frac{\delta-\sqrt{ }\left(\delta^{2}+4 \eta\right)}{2} \\
B=-\frac{\left(T_{2}-T_{1}\right)+A S}{s^{2}}
\end{gathered}
$$

and

$$
\begin{aligned}
& \delta=\left[\begin{array}{ll}
2 \lambda \\
c s
\end{array}-\frac{q_{q, s}}{k}\right] \\
& \left.\eta=\left\{\frac{2 \lambda}{c s^{2}}\left(T_{2}-T_{1}\right)+\frac{\lambda}{k c} \frac{\mathrm{d} q_{r}}{\mathrm{~d} x_{s}}\right)\right\} .
\end{aligned}
$$

We note that the expressions for $A$ and $B$ as given by equation (9) and (10) reduces to those given by Goodman [1] if the radiation contribution is set equal to zero.

\section{The radiative heat flux}

The radiative heat flux for a gray non-scattering medium and for diffusc cmission and reflection is given by [4, 5]

$$
\begin{aligned}
q_{r, \tau}= & 2 \pi C_{1} E_{3}(\tau)-2 \pi C_{2} E_{3}\left(\tau_{0}-\tau\right) \\
+ & 2 n^{2} \sigma \int_{0}^{\ddagger} T^{4}(t) E_{2}(\tau-t) \mathrm{d} t \\
& \quad-2 \mathrm{n}^{2} \sigma \int_{\tau}^{\tau_{0}} T^{-4}(t) E_{2}(t-\tau) \mathrm{d} t
\end{aligned}
$$

where

$$
\begin{aligned}
C_{1}=\frac{n^{2} \varepsilon_{1} \sigma T_{1}^{4}}{\pi}+2 \rho_{1} E_{3}\left(\tau_{0}\right) C_{2} \\
+\frac{2 \sigma \rho_{1} n^{2}}{\pi} \int_{0}^{\tau_{0}} T^{-4}(t) E_{2}(t) \mathrm{d} t
\end{aligned}
$$

$$
C_{2}=\frac{n^{2} \varepsilon_{2} \sigma T_{2}^{4}}{\pi}+2 \rho_{2} C_{1} E_{3}\left(\tau_{0}\right)
$$

$$
+\frac{2 \sigma \rho_{2} n^{2}}{\pi} \int_{0}^{\tau_{0}} T^{4}(t) E_{2}\left(\tau_{0}-t\right) \mathrm{d} t
$$

Equations (3), (4) and (7) require that the radiative heat flux* with its special and time derivatives be taken at $x=0$ and at $x=s$. Performing these operations results in the following integral expression for the propagation of the solid-liquid interface with time.

$$
t=\int_{0} \frac{[(t))}{\alpha\left[2\left(T_{2}-T_{1}\right)+A s-\frac{\lambda}{c}+\frac{1}{3}\left(T_{2}-T_{1}\right)-\frac{s^{2} \mathrm{~d} A}{6} \frac{A}{\mathrm{~d} s}-\frac{A s}{3}\right] s \mathrm{~d} s} .
$$

Equation (16) in the absence of radiation yields the following closed form solution presented in [1].

$$
s=W \sqrt{ } t
$$

where

$$
\frac{W}{2 \sqrt{\alpha}}=(\sqrt{3})\left[\frac{1-(1+\mu)^{\frac{1}{2}}+\mu}{5+(1+\mu)^{\frac{1}{2}}+\mu}\right]^{\frac{1}{2}}
$$

and

$$
\mu=\frac{2\left(T_{2}-T_{1}\right) c}{\lambda}
$$

The solution to equation (16) requires that the radiative heat flux and its derivatives be determined first. That involves integration over the temperature distribution in the solid which is unknown a priori and demands values for $A$ and $B$ that reflect the effect of the radiative transport at each value of $s$. The method of successive substitution, then, was used to determine $A$ and $B$ from equations (9) (12). When the difference in the calculated values of $A$ and $B$ between two consecutive iterations was within 1 per cent of the magnitude of each, the iteration is stopped and the values then obtained were considered satisfactory for the particular value of $s$. The method converged to the correct values of $A$ and $B$ very fast and in most cases did not require more than two iterations. With the correct values of $A$ and $B$ and the corresponding values for $q_{r, s}, q_{r, 0}$ and $\mathrm{d} / \mathrm{d} x\left[q_{r}\right]_{s}$, equation (16) was, then, integrated numerically to obtain the value of time for that chosen $s$. The method was repeated for each prescribed value of $s$ resulting in the desired $s$ vs. $t$ curve and in the temperature distribution at each $s$.

\section{RESULTS AND CONCLUSIONS}

Figure 1 shows the location of the solid-liquid interface as a function of time with and without the effects of radiation

* In the radiative heat tlux the exponential kernel approximation was used namely $E_{2}(t) \approx \frac{3}{4} \mathrm{e}^{-\frac{3}{2} t}$. 


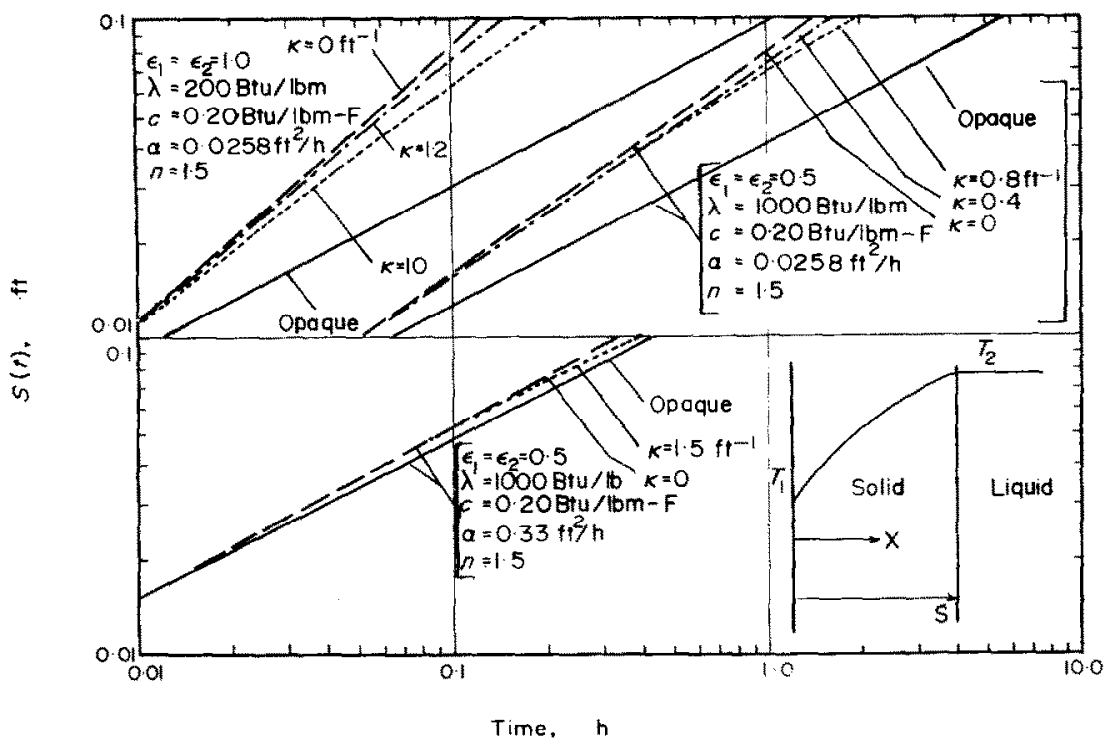

FIG. 1. Thickness of solid vs. time. $\left(T_{1}=1800^{\circ} \mathrm{R}, T_{2}=2000^{\circ} \mathrm{R}\right)$

for three sets of conditions. Results for other thermophysical properties behaved in a similar fashion. It can be seen that

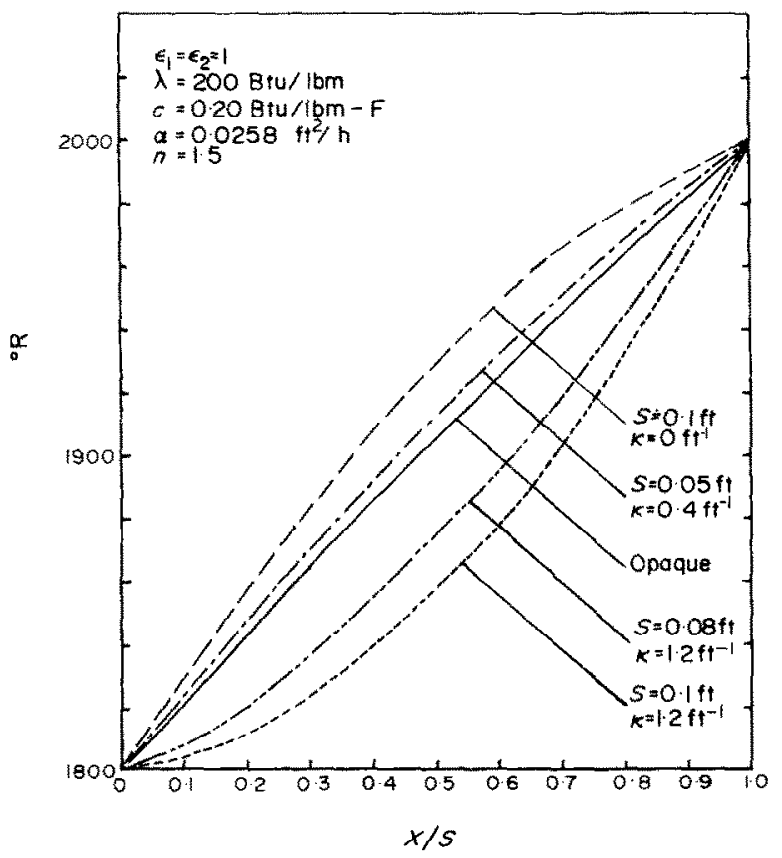

Fig. 2. Temperature distribution in the solid. the case of the nonparticipating medium $*=0$ represents an upper bound on the solidification rate while the pure conduction case (opaque body) represents a lower bound. An increase in the radiation absorption in the medium shifts the solidification curve towards the pure conduction case. In Fig. 2 we see that the radiation phenomena appreciably affects the temperature distribution in the solid phase. For the present model the temperature distribution for a nonparticipating medium is higher than that for a pure conducting one. This might seem at first unusual, however, it should be emphasized that the solidification rate for $\kappa=0$ is much higher than that for pure conduction. Increasing the absorption in the medium drops the temperature distribution ultimately below that of pure conduction. This can be attributed to a net radiation loss by the medium to the boundary. The propagation rate of the interface and the temperature distribution in the solid phase for materials with high thermal diffusivities were little affected by the radiation contribution.

\section{REFERENCES}

1. T. G. Conoman, The heat balance integral and its application to problems involving a change of phase, Trans ASME, 335-342 (Feb. 1958).

2. H. G. LANDAU, Heat conduction in melting solid, $Q$. Appl. Math. 18, 81 (1950).

* For a nonparticipating medium the radiative heat flux is constant for all values of $s$ and reduces to the case of the gray parallel plates. 
3. R. GreiF and A. E. KaSSEM, Heat transfer in a growing vapor film, Z. Agnew. Math. Phys. 19(5), 824 (1968).

4. T. J. Love, Radiative Heat Transfer. Charles E. Merrill (1968).

5. E. M. SPARRow and R. D. Cess, Radiation Heat Transfer. Brooks/Cole (1966).

6. P. L. Chambre, On the dynamics of phase growth. $Q . J$. Mech. Appl. Math. IX, pt. 2, 224233 (1956).
7. M. Elmas, On the solidification of a warm liquid flowing over a cold wall, Int. J. Heat Mass Transfer 13.1060-1062 (1970).

8. H. S. CARSLAW and J. C. JAEGaR, Conduction of Heat in Solids. Oxford (1959).

Int. J. Heat Mass Transfer. Vol. 14, pp. 2164-2165. Pergamon Press 1971. Printed in Great Britain

\title{
HEAT TRANSFER MECHANISM IN RECIRCULATING WAKES
}

\author{
JACOB H. MASLIYAH
}

Chemical Engineering Dept., University of British Columbia, Vancouver, B.C., Canada

(Received 12 March 1971)

\section{NOMENCLATURE}

$a$, equatorial radius of oblate spheroid:

$c_{p}$, thermal capacity :

$h$, local heat transfer coefficient:

$k$, thermal conductivity :

$N u(\eta), \quad$ local Nusselt number $=2 a h / k$;

Pr, $\quad$ Prandtl number, $c_{p} \mu / k$ :

Re, $\quad$ Reynolds number $=2 a \rho U / \mu$;

$T$, normalized local temperature:

$U$, dimensional velocity of the undisturbed fluid at infinity :

$v, \quad$ dimensionless local velocity $=v^{\prime} / U$ :

$v^{\prime}$, dimensionless local velocity of the fluid.

\section{Greek letters}

$\eta, \quad$ spheroidal coordinates, angle:

$\mu, \quad$ viscosity:

$\xi, \quad$ spheroidal coordinate orthogonal to $\eta$ :

$\rho$, density;

$\psi, \quad$ dimensionless stream function $=\psi^{\prime} / a U^{2}$ :

$\psi^{\prime}$ dimensional stream function.

$$
\begin{array}{cl}
\text { Subscripts } & \\
a, & \text { spheroidal surface: } \\
\eta, & \eta \text {-direction } \\
\xi, & \xi \text {-direction. }
\end{array}
$$

THE FLOW of a Newtonian incompressible fluid past an oblate spheroid at intermediate Reynolds number $(\simeq 1 \mathrm{~W})$ is characterized by the appearance of a steady axisymmetric recirculating wake which is attached to the downstream side of the spheroid, Rimon and Lugt [1] and Masliyah and Epstein [2].

When the spheroid surface is at a higher temperature than the ambient fluid, heat is transferred to the fluid. The role of the wake in the heat transfer is best demonstrated by the solution of the equations governing the fluid motion and the heat transfer.

The energy equation for forced convection with constant fluid properties, in oblate spheroidal coordinates $(\xi, \eta)$ is

$$
\begin{aligned}
v_{\xi} \frac{\partial T}{\partial \xi}+v_{\eta} \frac{\partial T}{\partial \eta}=\frac{2 \cosh \xi_{a}}{\operatorname{Pr} \cdot \operatorname{Re}\left(\cosh ^{2} \xi-\sin ^{2} \eta\right)^{\frac{1}{2}}} & \left(\frac{\partial^{2} T}{\partial \xi^{2}}+\frac{\partial^{2} T}{\partial \eta^{2}}+\cot \eta \frac{\partial T}{\partial \eta}+\tanh \frac{\partial T}{\partial \xi}\right) .
\end{aligned}
$$

The velocity components $v_{\xi}$ and $v_{\eta}$ are given by the numerical solution of the momentum equations for steady axisymmetric flow [2]. The boundary conditions employed are $T=1$ at the surface of the spheroid $\left(\xi=\xi_{a}\right), T=0$ at $\xi \rightarrow \infty$ and $\partial T / \partial \eta=0$ at $\eta=0$ and $\pi$. The solution of the energy equation was accomplished by using central finite difference equations, Masliyah and Epstein [3].

The closed recirculating streamlines inside the wake, together with the isotherms, as obtained by the complete solution of the motion and energy equations reveal a rather interesting mechanism of heat transfer. The fluid in the wake enclosed by the streamline $\psi=0$ in Fig. 1 does not leave the recirculating wake, by definition of a streamline. It follows that the heat emanating from that portion of the spheroid surface surrounded by the wake is ultimately transferred by conduction across the streamline $\psi=0$. A hot element of fluid situated near the surface on one of the closed streamlines, say $\psi=-0.01$, loses its heat on its 